\title{
La práctica de la traducción inversa hacia la lengua alemana en España: un estudio sociológico
}

\author{
Lorena Pérez Macías \\ Universidad Pablo de Olavide \\ ipermac@upo.es
}

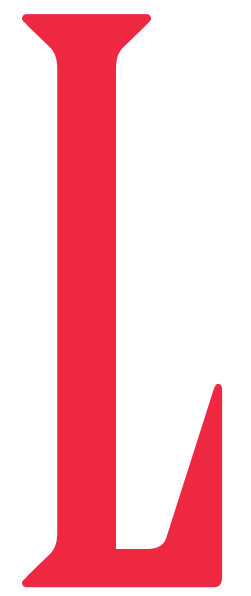

a traducción a la lengua no nativa del traductor (también conocida como traducción inversa en español o HinÜbersetzung en alemán) es la variedad de traducción que tradicionalmente ha recibido una menor atención en las publicaciones sobre Traductología, ya que se había asumido que solo existía una dirección «normal», aquella en la que el traductor traduce exclusivamente a su lengua materna (Corpas, 2001). Incluso, como indica Huang (2014), llegando a cuestionar en algunos casos la posibilidad o la existencia de este tipo de traducción. No obstante, cabe destacar que, con el paso del tiempo, el estudio de la direccionalidad en general y de la traducción inversa en particular ha ido cobrando una relevancia cada vez mayor dentro de la Traductología (Pérez, 2013), debido, en gran parte, a la evolución de esta práctica en el sector profesional de la traducción, donde parece estar cobrando de forma paulatina un mayor peso. La existencia de este tipo de traducción es una realidad profesional que no se puede obviar actualmente, aunque su práctica aún no esté generalizada entre la mayoría de los profesionales del sector (Gallego, 2014).

El objetivo de este estudio es mostrar la realidad en torno a la práctica de la traducción inversa hacia la lengua alemana en el sector profesional de la traducción en España. Para ello, se presentará una muestra parcial de los datos recopilados a través de un cuestionario que formaba parte de un estudio más amplio realizado dentro de un programa de Doctorado de la Universidad Pablo de Olavide.

\section{Breve aproximación histórica}

La traducción a una lengua extranjera era una práctica común ya desde la Antigua Grecia y la cooperación entre traductor nativo y no nativo estaba ya presente en la teoría de la traducción de la Biblia (Pokorn, 2003: 122). Antes del Renacimiento (siglo XVI), las traducciones se realizaban casi de manera indiferente en ambas direcciones e, incluso, era más habitual realizarlas a la lengua extranjera, pero fue a partir de este punto cuando empezó a plantearse una disyuntiva, hasta que finalmente se llegó a la afirmación de la superioridad de la traducción directa (Guatelli-Tedeschi y Le Poder, 2003: 273). La figura de Martin Luther (14831546) destaca por plantear, por primera vez, la superioridad de la traducción directa. Tras él, Wilhelm von Humboldt (1767-1835), seguidor del primero, defendió esta misma idea. Von Humboldt afirmaba que cada lengua posee sus propios mecanismos para expresar unas determinadas ideas y que estos mecanismos solo eran accesibles a las personas que hablaban una lengua desde su nacimiento (Chesterman, 1998: 62). En España, cabe destacar el caso de la Escuela de Toledo (siglos XII y XIII), donde 


\section{Resumen:}

La traducción a la lengua no nativa del traductor es la variedad de traducción que tradicionalmente ha recibido una menor atención. El objetivo de este estudio es describir la realidad en torno a la práctica de la traducción inversa hacia la lengua alemana en el sector profesional de la traducción en España. Para ello, se presentarán los principales resultados a través de un cuestionario online administrado a traductores españoles en ejercicio, de los cuales, un porcentaje significativo trabaja con la lengua alemana. A través de este método de recopilación de datos, se pretende conocer la opinión de la muestra sondeada respecto a cuestiones como la formación o las tarifas en relación con la traducción inversa, entre otras.

Palabras dave: traducción inversa; direccionalidad; mercado laboral; cuestionario

\section{Zusammenfassung:}

Die Übersetzung in die Nicht-Muttersprache des Übersetzers ist die Vielfalt der Übersetzungen, der traditionell am wenigsten Beachtung gefunden hat. Ziel dieser Studie ist es, die Realität der Praxis der Rückübersetzung ins Deutsche im Bereich der professionellen Übersetzung in Spanien zu beschreiben. Zu diesem Zweck werden die wichtigsten Ergebnisse durch einen Online-Fragebogen präsentiert, der an praktizierende spanische Übersetzer gerichtet ist, von denen ein erheblicher Prozentsatz auf Deutsch arbeitet. Mit dieser Methode der Datenerhebung soll unter anderem die Meinung der Befragten zu Themen wie Schulungen oder Gebühren im Zusammenhang mit der Rückübersetzung ermittelt werden.

Schlüsselwörter: Rückübersetzung; Direktionalität; Arbeitsmarkt; Fragebogen se traducían textos con el fin de trasladar la sabiduría de Oriente a Europa. Cabe destacar aquí la forma en la que se realizaban estas traducciones, puesto que trabajaban en grupos de dos colaboradores: uno de ellos traducía de forma directa y el otro de manera inversa (Wimmer, 2011: 14-15). Fuera de Europa, destaca el caso de China, donde, durante el siglo II a.C., se llevaron a cabo las primeras traducciones de los textos sagrados del budismo de forma inversa (Hurtado, 1996: 59).

Hace medio siglo aproximadamente, el prestigio con el que contaban los traductores que eran capaces de realizar traducciones inversas era muy diferente al que obtendrían hoy en día por la misma labor. En aquel entonces, los traductores que podían traducir desde su lengua materna hacia un idioma extranjero eran los más cotizados, ya que se les presuponía haber adquirido el nivel máximo de competencia en ese idioma. Llegaban a cobrar incluso un porcentaje adicional por tratarse de una traducción hacia otra lengua, ya que se tenía en cuenta la dificultad añadida que conlleva una tarea de esta envergadura (Martorell, 2012).

\section{La prática de la traducción inversa actualmente: factores influyentes}

A continuación, se van a presentar una serie de factores que influyen de una forma directa en la práctica profesional de la traducción a una lengua no nativa. Como punto de partida, se resumirán los factores recogidos en Kelly et al.
(2003: 51-59) y, a partir de ahí, se completarán con otras referencias:

- El mercado profesional: es el que finalmente propicia que este tipo de traducción se pueda llevar a cabo. Un ejemplo de esto sería lo que ocurre en los países anglófonos, en los que existe una postura más claramente en contra de la traducción inversa. Pero incluso en los lugares donde está generalizada y aceptada esta práctica, se recurre a ella como opción menos deseable, normalmente por falta de traductores nativos de esa lengua a la que se quiere traducir.

- Tipología de textos: se obtienen buenos resultados en textos muy especializados, ya que en este caso lo más importante es la comprensión del original. Si se presta atención a la función del texto, los textos con una función expresiva no serían idóneos para este tipo de traducción. Durán (2012: 110-111), por otra parte, dedica un apartado en su obra a la traducción inversa dentro de una tipología concreta de textos: la traducción turística. En este caso, destaca la mala calidad, por regla general, de las traducciones, ya que son realizadas principalmente por hablantes no nativos de la lengua hacia la que se realiza la traducción. No obstante, señala que, cada vez más, son revisadas posteriormente por nativos de esa lengua, por lo que el nivel de calidad va aumentando progresivamente.

- El grado de elaboración del producto: desde el punto de vista del cliente, lo que se pretende es que 
el producto final funcione adecuadamente en la nueva situación comunicativa para la que se realizó. Tener en cuenta este factor permite, por ejemplo, modificar el precio del encargo según el grado de elaboración que se necesite. En Kelly et al. (2003: 55) se describen varias clasificaciones en relación con este factor según distintos autores: Gouadec, por ejemplo, en 1989, distingue entre a) nivel 1 o calidad revisable, b) nivel 2 o calidad entregable y c) nivel 3 o calidad para difusión o publicable; Schopp, por su parte, en 1995, diferencia entre a) traducción en bruto, b) traducción lista para maquetar y c) traducción lista para imprimir, e indica que el nivel «traducción en bruto» es el que se le puede exigir a una traducción inversa.

- Laética personal del traductor: un punto importante en la formación de los futuros traductores es hacerles capaces de decidir hasta qué punto pueden cumplir un proyecto que se les encomienda y que reflexionen sobre a) si son capaces por sí mismos de realizar una traducción en la dirección y con el grado de elaboración requeridos, b) si su traducción requerirá una posterior revisión y c) si su nivel de competencia traductora hacia la lengua extranjera para el encargo en cuestión recomienda no aceptarlo. Respecto a este último punto, Roiss (2016) se basa en las subcompetencias de Kelly (2002) para analizar los errores más comunes de los alumnos en cuanto a la traducción inversa de textos administrativos de español a alemán. Por citar algún ejemplo de este estudio, la autora, en referencia a la subcompetencia temática, es decir, aquella que comprende los conocimientos básicos sobre los campos temáticos en los que trabaja el traductor (Kelly, 2002: 14), presenta una serie de errores que se repiten entre los alumnos y les ofrece unas cuestiones para evitarlos. Estos errores, llevados al ámbito profesional, podrían servir para entender la inseguridad que pueden sentir los traductores profesionales al enfrentarse a un encargo de traducción inversa especializada hacia el alemán:

- Sensibilisierung im Bereich der Synonyme [...]: Wir haben gesehen, dass zwei Komposita mit dem Anfangselement Geburts- gebildet werden können: Geburtsanzeige / Geburtsurkunde. Handelt es sich dabei um Synonyme? [...]

- Kompositabildung ohne Anwendung thematischen Wissens [...]: Wurde für die nachstehende Übersetzung des Titels das nötige thematische Wissen von dem Übersetzer mit Deutsch als Muttersprache in Betracht gezogen? Cuestionario para la Declaración de Nacimiento en el Registro Civil - Formular für die Geburtsurkunde des Zivilstandsamts. Wie muss es richtig heißen und warum? [...]. (Roiss, 2016: 249).

- Precio: una traducción inversa requiere del traductor unas habilidades y capacidades diferentes a las necesarias para la realización de una traducción hacia la lengua materna del traductor. Todo esto influye en el precio, tanto desde la perspectiva del traductor como desde la del cliente. El traductor querrá cobrar teniendo en cuenta las horas y el esfuerzo que haya tenido que dedicarle a esa traducción; el cliente, si está debidamente informado (que sería la situación ideal), sabrá que la traducción realizada por un traductor no nativo será diferente a la que podría realizar un traductor que trabaje hacia su lengua materna y aceptará el riesgo de que su texto pierda, como mínimo, algunos matices culturales y aceptará un presupuesto que considere que tiene en cuenta estos riesgos.

- Lenguas de trabajo: debido al surgimiento del inglés como lingua franca, muchos textos traducidos hacia esta lengua no requerirán una traducción hacia una cultura específica, sino que irán dirigidos a un público internacional que abarque muchas culturas distintas para el que la lengua inglesa no será su lengua nativa. McAlester (1992: 292-293) llega incluso a afirmar que, en estos casos, un traductor que no tenga inglés como lengua materna se encontrará incluso en una mejor posición para realizar este tipo de traducciones que uno que sí tenga este idioma como lengua materna. Opdenhoff (2011), por su parte, plantea la relación existente entre las lenguas de trabajo y el nivel de producción. A este respecto, presenta la idea de que hay lenguas que se prestan más que otras a ser lenguas meta si trabajamos con ellas de manera inversa y este filtro funcionaría en base al grado de esfuerzo que conllevara la traducción, teniendo en cuenta las características específicas de las lenguas con las que trabajáramos. Para hacer más fácil la comprensión de este punto, citamos un ejemplo de Wilss (1978:346; cit. Opdenhoff, 2011:140-141) en el que se pueden visualizar las reestructuraciones morfosintácticas necesarias al traducir una frase alemana al inglés y al español: 


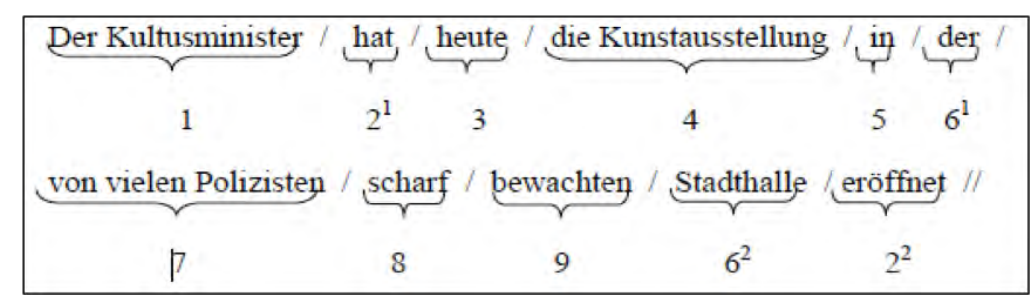

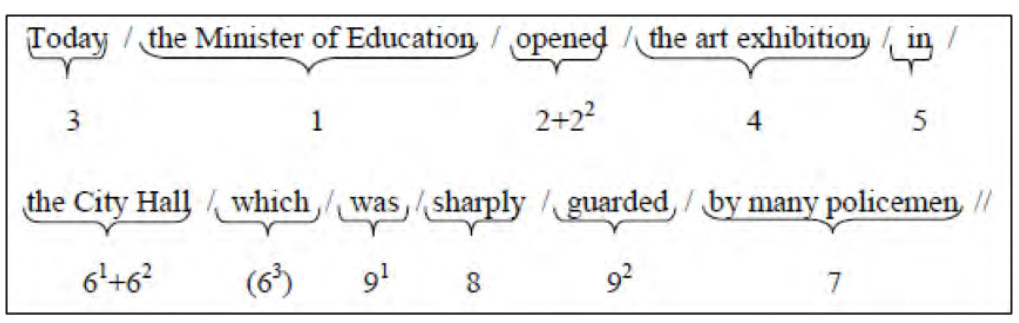

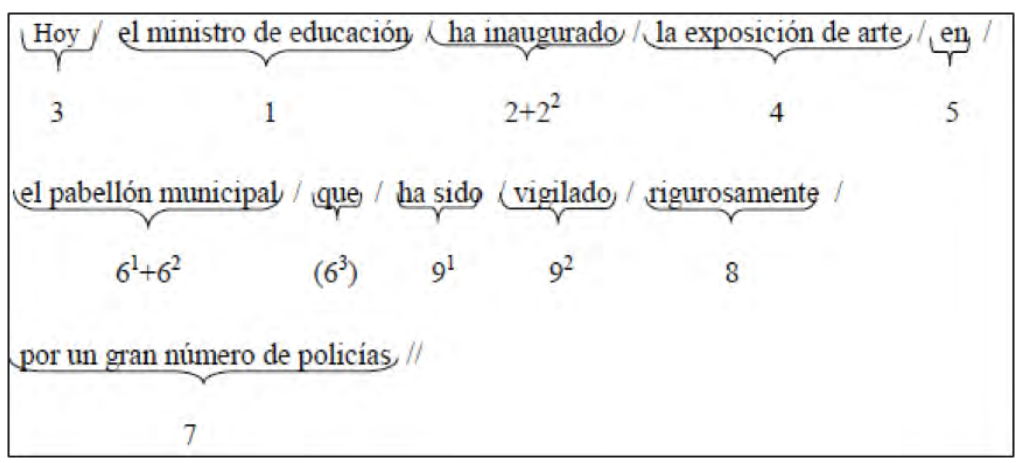

de la traducción en España se va a presentar, a continuación, una muestra parcial de los datos recopilados a través de un cuestionario en línea que formaba parte de un estudio más amplio realizado dentro de un programa de doctorado de la Universidad Pablo de Olavide, de Sevilla. Este estudio tenía como público objetivo a traductores profesionales en activo que ejercieran en España en el momento de la investigación. El enlace de acceso al cuestionario $^{1}$ se distribuyó a través de correo electrónico y redes sociales (muestreo no probabilístico) en dos fases diferenciadas en el tiempo: 2014 y 2018. No obstante, se mostrarán los resultados obtenidos en ambas fases de manera conjunta, puesto que en el caso del alemán no se han apreciado diferencias significativas entre los datos recopilados como consecuencia del paso del tiempo. Finalmente, participaron 118 sujetos en el estudio. Con el objetivo de resolver este estudio dentro de los límites que le corresponden, se analizarán aquellos aspectos que se hayan considerado más relevantes dentro del conjunto de resultados.

Desde una perspectiva cualitativa, es posible realizar un recorrido descriptivo de la situación actual en

Imagen 1. Lengua origen: alemán

Imagen 2. Lengua meta inglés

Imagen 3. Lengua meta español

Si nos fijamos en la estructura sintáctica de la versión inglesa y de la española podemos apreciar que son prácticamente iguales, mientras que, por otro lado, ambas distan mucho de la estructura que presenta la frase en alemán. De este ejemplo podemos deducir que, presumiblemente, será más factible realizar una traducción inversa al inglés que al alemán partiendo de un texto escrito en español, ya que requerirá un menor esfuerzo para llevarla a cabo y habrá un menor riesgo de cometer errores al haber menos diferencias sintácticas.

\section{Resultados del estudio de campo}

Con el fin de conocer la realidad respecto a las percepciones en torno a la práctica de la traducción inversa hacia la lengua alemana en el sector profesional

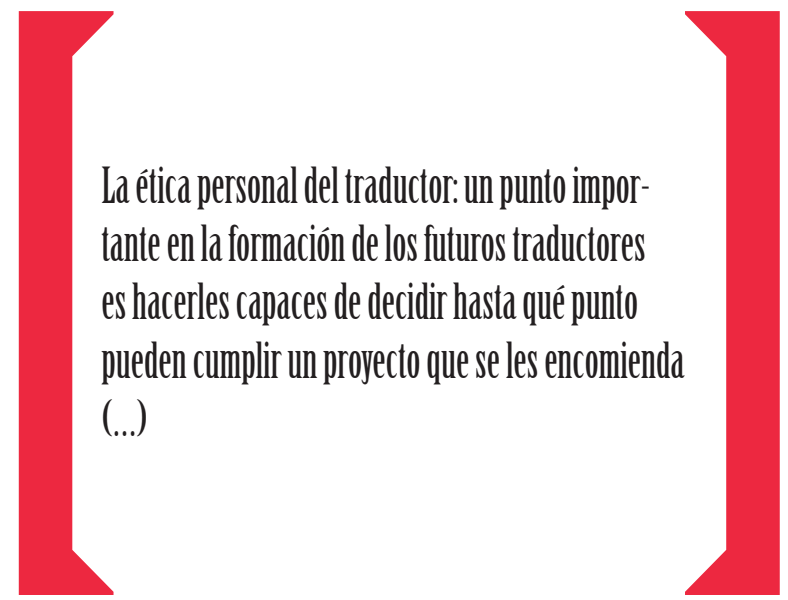

el mercado de la traducción inversa de español a alemán con los datos obtenidos. Para ello, se compararán estos datos, además, con algunos recopilados en estudios con cuestionarios que han servido como antecedentes para la realización de esta investigación y que se detallan en una 


\begin{tabular}{|l|l|l|l|}
\hline \multicolumn{1}{|c|}{ Autor/es } & \multicolumn{1}{|c|}{ Temática estudio } & \multicolumn{1}{c|}{ Población } & \multicolumn{1}{c|}{ Muestra } \\
\hline Roiss (2001) & $\begin{array}{l}\text { El ejercicio de la } \\
\text { traducción inversa } \\
\text { en España. }\end{array}$ & $\begin{array}{l}\text { Traductores profesionales miembros de APETI, } \\
\text { antiguos alumnos de la Facultad de Traducción y } \\
\text { Documentación de la Universidad de Salamanca } \\
\text { y agencias de traducción de Madrid, Barcelona y } \\
\text { Valencia. }\end{array}$ & $\begin{array}{l}\text { Muestreo no } \\
\text { probabilístico. } \\
330 \text { sujetos. }\end{array}$ \\
\hline Zimmermann (2007) & $\begin{array}{l}\text { El ejercicio de } \\
\text { la traducción } \\
\text { especializada } \\
\text { inversa en España. }\end{array}$ & $\begin{array}{l}\text { Alumnos que cursaran o hubieran cursado } \\
\text { asignaturas de Traducción Especializada Inversa } \\
\text { español-alemán en algunas de las seis universidades } \\
\text { españolas escogidas. }\end{array}$ & $\begin{array}{l}\text { Muestreo no } \\
\text { probabilístico. } \\
54 \text { sujetos. }\end{array}$ \\
\hline Gallego (2014) & $\begin{array}{l}\text { El ejercicio de } \\
\text { la traducción } \\
\text { especializada } \\
\text { inversa en España. }\end{array}$ & $\begin{array}{l}\text { Traductores jurados que, al menos alguna vez, } \\
\text { hubieran trabajado hacia el alemán, el francés o el } \\
\text { inglés. }\end{array}$ & $\begin{array}{l}\text { Muestreo no } \\
\text { probabilístico. } \\
500 \text { sujetos. }\end{array}$ \\
\hline
\end{tabular}

Tabla 1. Relación de antecedentes de estudios de estudios con encuesta centrados en el ejercicio de la traducción inversa en España

Teniendo en cuenta estos antecedentes es posible apreciar que no existen apenas estudios actualizados sobre esta temática, por lo que el estudio que aquí se presenta pretende comprobar, asimismo, si la práctica de la traducción inversa o el perfil de quienes la realizan ha evolucionado en los últimos años, en aquellos casos en los que los datos obtenidos se puedan comparar con los recopilados en estudios anteriores.

Según los datos recogidos a través del cuestionario, destaca que, aproximadamente, el $80 \%$ de los encuestados había realizado traducciones inversas hacia la lengua inglesa, el $24 \%$ hacia el francés y el $10 \%$ había realizado traducciones inversas hacia la lengua alemana. Cabe señalar que, en algunos casos, los encuestados indicaron realizar traducciones inversas hacia varias lenguas.

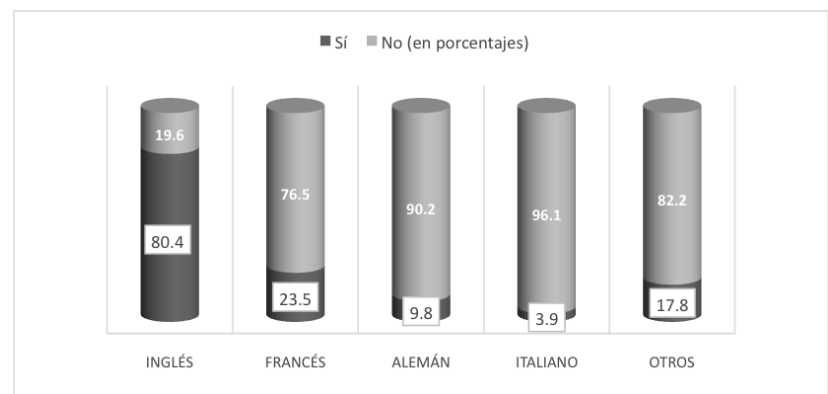

Gráfico 1. Lenguas de las traducciones inversas lengua alemana, ya que resulta especialmente relevante su experiencia en traducciones inversas para este estudio. A continuación, se presentan los principales resultados obtenidos en relación con el perfil de los profesionales que realizan traducciones inversas al alemán y algunas cuestiones generales sobre la práctica de este tipo de traducciones:

- Lenguas de trabajo: en relación con las lenguas de trabajo de esta parte de la muestra, de los diez sujetos en total que trabajaban hacia la lengua alemana, seis tenían alemán como primera lengua extranjera y cuatro como segunda lengua extranjera. Este dato parece mostrar que no hay demasiada diferencia entre los sujetos que poseen alemán como primera lengua extranjera y los que la tienen como segunda respecto a la aceptación de proyectos de traducción inversa al alemán o no.

- Formación: ocho sujetos indicaron haber estudiado la Licenciatura en Traducción e Interpretación y dos Filología. Parece apreciarse una clara evolución en el perfil del traductor respecto a su formación académica, ya que el estudio de Roiss (2001) la mayoría de los sujetos habían cursado Filología, debido a que la implantación oficial de los estudios de Traducción e Interpretación en España se había llevado a cabo hacía relativamente poco tiempo (en 1992). 
- Años de experiencia en el sector: seis encuestados señalaron tener entre seis y diez años de experiencia y cuatro encuestados tenían entre dos y cinco años de experiencia. Si comparamos estos datos con los recopilados por Gallego (2014), es posible observar que están en la misma línea, puesto que la mayoría de los sujetos de su estudio tenían menos de cinco años de experiencia en el sector, mientras que, en segundo lugar, se encontraban aquellos que contaban con una experiencia de entre cinco y diez años. Teniendo en cuenta estos datos, parece continuar la tendencia de no realización de inversas entre la nueva generación de traductores recién incorporados al mercado laboral, ni entre aquellos que tienen más de diez años de experiencia, quizás debido, en el primero de los casos, a la falta de seguridad del profesional en sus primeros años de ejercicio $y$, en el segundo, a la mejor consciencia de la dificultad que puede entrañar esta tarea tras años de experiencia.

- Situación laboral: seis encuestados indicaron trabajar en plantilla (dos para una empresa que no pertenece al sector de la traducción y cuatro en empresas de servicios lingüísticos) y cuatro encuestados señalaron trabajar como autónomos directamente con clientes que requerían sus servicios. Si se comparan estos datos con los recopilados por Gallego (2014), se puede apreciar una evolución en cuanto a la situación laboral de aquellos profesionales que realizan traducciones inversas, ya que, en el estudio de este autor, la mayoría de estos trabajaba como profesional autónomo $\mathrm{y}$, en segunda posición, en empresa ajena a la traducción. Quizás esto se deba a la combinación español>alemán en la que se ha centrado nuestro estudio, cuya realización podría deberse más a una imposición por parte de la empresa en el caso del trabajo por cuenta ajena que a una decisión propia, como podría ser el caso de los profesionales autónomos.

- ¿Cree que recibió la formación académica necesaria para realizar traducciones inversas de una calidad aceptable?: todos los sujetos indicaron que no. En cuanto a las justificaciones que aportan los participantes para explicar el motivo por el cual no se sienten con la suficiente formación, la que más se repite entre los sujetos que sí han recibido una formación específica en Traducción es la queja de que se le dedica muy pocas horas a este tipo de traducción en la formación universitaria, en comparación con las que se le dedica a la traducción directa. Aunque no es posible contar con datos relativos a esta pregunta en otros estudios, Roiss (2001) incluye una pregunta abierta en su cuestionario que puede ayudar a complementar esta información: Todas las Facultades de Traducción en España ofrecen asignaturas de traducción del español hacia una lengua extranjera. ¿Cree Ud. Que tiene razón de ser, o el único fin de impartir este tipo de clases consiste en la mejora de la lengua extranjera, ya que no se debe traducir a un idioma que no es el materno? La mayoría de los sujetos de este estudio se mostraron a favor de la práctica de la traducción inversa y a su inclusión en los planes de estudios universitarios, por lo que no parece ser una idea nueva la necesidad de incluir la práctica de la traducción inversa de manera eficiente y útil en la formación universitaria de los futuros traductores, aunque como se puede apreciar gracias a los datos más actuales, esta formación no parece aún resultar suficiente.

- ¿ ¿Con qué frecuencia realiza traducciones inversas?: todos indicaron que únicamente las realizan de manera puntual, cuando no pueden rechazar un encargo (cliente habitual, compromiso...). No obstante, resulta relevante tener en cuenta aquí los datos relativos a la frecuencia general de la traducción inversa que Gallego (2014) incluye en su estudio. En este estudio, la mayoría de los encuestados realizaba traducciones inversas a menudo, muy a menudo o siempre, quizás debido al público objetivo concreto de esa investigación, compuesto por traductores jurados. En el estudio llevado a cabo por Roiss (2001), por el contrario, sí se mantiene la línea mostrada en los datos más actuales, ya que para la mayoría de los encuestados la traducción inversa constituía alrededor de un 10 $\%$ del volumen total de su trabajo.

- ¿Han sido revisadas posteriormente sus traducciones inversas por un corrector o revisor cuya lengua nativa sí era la lengua meta de la traducción?: seis de los encuestados indica que sí han sido revisadas, dos que no y otros dos 
señalan la opción «NS/NC». Entre los comentarios aportados destacan que en la mayoría de los casos ellos mismos buscan al corrector/revisor nativo, mientras que en uno de los casos es el propio cliente quien se encarga de esto. El resto de las respuestas indican que no tienen un protocolo de actuación definido, sino que depende del texto, de la empresa, etc. Estos datos son similares a los recopilados por Roiss (2001), ya que la mayoría de los participantes de su estudio confirmaron dejar que un hablante nativo revisara su traducción a posteriori.

\section{Conclusiones}

Gracias a los resultados obtenidos en este estudio, es posible constatar el predominio de la lengua inglesa en las tareas de traducción inversa. No obstante, existe también un porcentaje de profesionales que, sin ser nativos de lengua alemana, realizan traducciones inversas hacia esa lengua. El perfil de estos profesionales parece coincidir, en gran medida, en varios aspectos significativos: poseer un título universitario en Traducción o Filología, contar con más de seis años de experiencia y trabajar en plantilla en una empresa (ya sea de servicios lingüísticos o en algún sector ajeno al ámbito de la traducción). En todos estos casos hay mayoría de encuestados que cumplen esos criterios.
La opinión de los encuestados respecto a la formación académica recibida parece coincidir, ya que todos aluden a la falta de formación en este ámbito durante la etapa universitaria y la mayoría considera que su competencia lingüística y profesional no es la adecuada para realizar este tipo de tareas. Por ello, solo aceptan encargos de este tipo en situaciones puntuales e impuestas en la mayoría de los casos. No obstante, sí parece haber un mayor acuerdo en la concienciación de que una traducción inversa no puede llegar al nivel de una traducción directa sin una posterior revisión/corrección realizada por un profesional nativo de esa lengua. En este caso, sí se percibe una mayor aceptación entre los profesionales de la traducción.

Como se puede apreciar por toda la información anterior, la traducción inversa y, en concreto, la traducción inversa hacia el idioma alemán, es una actividad que está cobrando de forma paulatina un mayor peso dentro del mercado laboral de la traducción. A pesar de que la teoría y la práctica sobre este tema no van al mismo ritmo, es evidente que la existencia de este tipo de traducción es una realidad profesional hoy en día que no se puede obviar. Asimismo, se puede afirmar que la traducción hacia una lengua extranjera posee unas características propias que la diferencian de la traducción directa y que son necesarias que el profesional de la traducción conozca para poder aceptar o no un encargo de esta envergadura.
Bibliografia

Chesterman, A. (1998): “Causes, Translations, Effects", Target 10 (2), 201-230.

Corpas, G. (2001): "Compilación de un corpus ad hoc para la enseñanza de la traducción inversa especializada, en TRANS. Revista de Traductología 5248 (163), 155-184.

Durán, I. (2012): “Caracterización de la traducción turística: problemas, dificultades y posibles soluciones", en Revista de Lingüística $y$ Lenguas Aplicadas, (7), 103-113.

Gallego, D. (2014): "A vueltas con la traducción inversa especializada en el ámbito profesional. Un estudio basado en encuestas", en TRANS. Revista de Traductología 8, 229-238.

Guatelli-Tedeschi, J. y Le Poder, E. (2003): "De una direccionalidad distorsionada", en Kelly, D., Martin, A., Nobs M. L., Sánchez, D. y Way, C. (eds.), La direccionalidad en traducción e interpretación: perspectivas teóricas, profesionales y didácticas, Granada, Atrio, 267286.

Huang, W. (2014): 'Una propuesta comunicativa para la traducción pedagógica del chino al español en China, tesis doctoral dirigida por la Dra. Allison Beeby, Barcelona, Universitat Autònoma de Barcelona.

Hurtado, A. (1996): La enseñanza de la traducción, Castelló, Publicacions de la Universitat Jaume I.
Kelly, D. (2002): "Un modelo de competencia traductora: bases para el diseño curricular", en Puentes 1, 9-20.

Kelly, D., Martin, A., Nobs M. L., Sánchez, D. y Way, C. (eds.) (2003): La direccionalidad en traducción e interpretación. Perspectivas teóricas, profesionales y didácticas, Granada, Atrio

McAlester, G. (1992): “Teaching translation into a foreign language $\_$status, scope and aims", en Dollerup, C. y Loddegaard, A. (eds.) Teaching translation and interpreting, Amsterdam \& Philadelphia, John Benjamins, 291-297.

Martorell, A. (2012): “Inversas (I)", en El Trujamán. Revista diaria de traducción [en línea] [http://cvc.cervantes.es/trujaman/ anteriores/septiembre 12/10092012.htm].

Opdenhoff, J. H. (2011): Estudio sobre la direccionalidad en interpretación de conferencias: de las teorías a la práctica profesional, tesis doctoral dirigida por la Dra. Presentación Padilla y el Dr. Francisco Díaz, Granada, Universidad de Granada.

Pérez, L. (2013): La situación actual de la traducción inversa en el mercado laboral: estudio de campo, trabajo de investigación inédito de acceso a Doctorado, tutelado por la Dra. Elisa Calvo, Sevilla, Universidad Pablo de
Olavide.

Pokorn, N. (2003): "The (in)competence of a native speaker in translation theory and practice", en Kelly, D., Martin, A., Nobs M. L., Sánchez, D. y Way, C. (eds.), La direccionalidad en traducción e interpretación: perspectivas teóricas, profesionales y didácticas, Granada, Atrio, 117-138.

Roiss, S. (2001): "El mercado de la traducción inversa en España, un estudio estadístico", en Hermēneus. Revista de Traducción e Interpretación 3, 397-408

Roiss, S. (2001): "Fehleranalyse im Hinübersetzungsunterricht (Spanisch-Deutsch) aus der Sicht der Motivationsforschung und des kompetenzorientierten Lernens", en Lebende Sprachen 61 (1), 242-257.

Wimmer, S. (2011): $\quad$ El proceso de la traducción especializada inversa: modelo, validación empírica y aplicación didáctica, tesis doctoral dirigida por el Dr. Wilhelm Neunzig, Barcelona, Universidad Autònoma de Barcelona.

Zimmermann, P. (2007): "Misión (casi) imposible. La traducción especializada inversa al alemán desde la mirada del alumno", en Santana, B. et al. (eds.), Puente entre dos mundos: últimas tendencias en la investigación traductológica alemán-español, 394-402. 